\title{
SIRT1 Is Reduced in Gastric Adenocarcinoma and Acts as a Potential Tumor Suppressor in Gastric Cancer
}

\author{
Yongguo Zhang Xiqiang Cai Na Chai Yong Gu Song Zhang \\ Meiling Ding Haichao Cao Sumei Sha Jipeng Yin Mengbin Li \\ Kaichun Wu Yongzhan Nie \\ State Key Laboratory of Cancer Biology and Department of Gastroenterology, \\ Xijing Hospital, The Fourth Military Medical University, Xi'an, Shaanxi Province, China
}

\section{Key Words}

Gastric adenocarcinoma $\cdot$ p53 $\cdot$ SIRT1 $\cdot$ Tumor suppressor

\begin{abstract}
Background: Silent mating type information regulation 2 homolog 1 (SIRT1) is a nicotinamide adenine dinucleotide-dependent deacetylase that is involved in a variety of biological processes. Recent studies have implicated the involvement of SIRT1 in tumorigenesis, but its role remains controversial even in the same tumor type. Summary: In this study, SIRT1 expression was examined in gastric adenocarcinoma by immunohistochemistry and quantitative real-time polymerase chain reaction. Cell proliferation, cell cycle, cell apoptosis and xenograft assays were performed to elucidate the roles of SIRT1 in gastric cancer. We found that SIRT1 expression was reduced in gastric adenocarcinoma at both the protein and mRNA levels. Tissue microarray analysis revealed a positive correlation between SIRT1 and p53 expression in gastric adenocarcinoma. Inhibition of SIRT1 by EX-527 or by shRNA-mediated silencing increased cell proliferation in vitro and in vivo. SIRT1 inhibition accelerated the G1-S phase transition and reduced apoptosis by regulating key factors involved in cell cycle control and apoptosis. On the contrary, the ectopic expression of SIRT1 or the activation of SIRT1 by resveratrol was sufficient to suppress the growth of gastric cancer cells in vitro and in vivo. Key Message: In our study, the ectopic expression or activation of SIRT1 arrested cells at the G1 phase by reducing the levels of Cdk4 and cyclin D1, and induced apoptosis by increasing the ratios of Bax/Bcl-2. Practical Implications: We define SIRT1 as a potential tumor suppressor in gastric cancer. Its activation or overexpression inhibits tumor proliferation in gastric cancer by modulating cell cycle and apoptotic pathway. Strategies to activate SIRT1 could be used to treat gastric cancer.
\end{abstract}


Zhang et al.: SIRT1 Is Reduced in Gastric Adenocarcinoma and Acts as a Potential Tumor Suppressor in Gastric Cancer

\section{Introduction}

Gastric cancer is the fourth most common cancer and the second leading cause of cancer-related deaths worldwide $[1,2]$. Although the prevalence and death rate related to gastric cancer have decreased worldwide, there are still 933,293 new cases and 700,000 deaths due to gastric cancer every year, and the 5-year survival rate for advanced-stage gastric cancer is approximately $20 \%[1,2]$. Therefore, it is urgent to discover novel mechanisms and targets involved in the development and progression of gastric cancer.

Recently, research has indicated that genetic stress may be involved in gastric carcinogenesis through $\mathrm{p} 53$, nuclear factor $\kappa \mathrm{B}$ or other pathways. Silent mating type information regulation 2 homolog 1 (SIRT1) is an important stress-responsive element that belongs to the class III histone deacetylases $[3,4]$. SIRT1 deacetylates histones and many non-histone proteins in a nicotinamide adenine dinucleotide $\left(\mathrm{NAD}^{+}\right)$-dependent manner and is involved in a variety of biological processes, including cell growth, apoptosis, adaptation to calorie restriction, organ metabolism and function, cell senescence and tumorigenesis [5-7].

Over the past decade, the function of SIRT1 in tumorigenesis has been studied. However, the role of SIRT1 as an oncogene or as a tumor suppressor remains controversial. Several studies have demonstrated that SIRT1 expression is higher in various human tumors, including gastric cancer, compared to the corresponding normal adjacent tissues [8-12]. SIRT1 deacetylates and inactivates a series of proteins involved in tumor suppression and DNA damage repair [13-16]. These findings support the potential role of SIRT1 as a tumor promoter. In contrast, other studies indicate that SIRT1 might act as a tumor suppressor, as its expression is reduced in many types of cancers [17-19]. Additional studies from transgenic mouse models have revealed that SIRT1 suppresses the development of spontaneous tumors and metabolic syndrome-associated cancers in multiple tissues $[18,20,21]$. Moreover, even for the same type of cancer, there are still conflicting reports about the expression of SIRT1. For instance, Sung et al. [22] found an overexpression of SIRT1 in breast cancer, whereas Wang et al. [17] reported a decreased expression in breast tumor. Stunkel et al. [10] reported that SIRT1 was highly expressed in colon carcinoma tissue samples, whereas Kabra et al. [19] showed SIRT1 expression to be silenced in a subset of colon cancer. SIRT1 expression was shown to be increased in human prostate cancer tissues [8]. However, deletion of SIRT1 resulted in the development of prostatic intraepithelial neoplasia lesions by repressing autophagy [23]. For hepatic carcinoma, Chen et al. [12] observed that SIRT1 was upregulated in a subset of hepatocellular carcinomas, whereas Wang et al. [18] found reduced SIRT1 expression in hepatic carcinoma. Therefore it would be necessary to further understand the real effect of SIRT1 in gastric cancer.

Cha et al. [9] found that SIRT1 is highly expressed in gastric cancer and is associated with the prognosis of gastric cancer patients. However, in this study we found that SIRT1 expression was decreased in gastric adenocarcinoma both at the protein and mRNA levels, and that its expression level was associated with the histological type of the tumor. Moreover, inhibition or knockdown of SIRT1 increased cell proliferation and tumor formation by regulating cell cycle progression and cell apoptosis, both in vitro and in vivo, whereas the activation or ectopic expression of SIRT1 inhibited gastric cancer cell proliferation and reduced tumor formation in nude mice. 


\section{Materials and Methods}

Tissue Samples and Immunohistochemical Staining

The gastric adenocarcinoma microarray was constructed from patient samples acquired from the Xijing Hospital of Digestive Diseases between 2008 and 2009 (Shanghai Biochip Co., Ltd.). A total of 112 human gastric adenocarcinoma tissues and the corresponding non-tumor tissues were included. Data on sex, age, histological type, TNM stage and metastasis were collected from the medical record library of the hospital. An additional 24 tumor tissue samples and the corresponding non-tumor mucosa tissue samples were collected from patients with gastric adenocarcinoma and immediately stored in liquid nitrogen after resection for quantitative real-time polymerase chain reaction (qRT-PCR) analysis. This study was approved by the Ethics Committee of the Fourth Military Medical University.

The tissue microarray was stained for SIRT1 and p53 expression as previously described. In brief, the tissue microarray was treated by microwave for $10 \mathrm{~min}$ for antigen retrieval. After blocking the endogenous peroxidase and non-specific staining, the tissue microarray was incubated with either of two anti-SIRT1 antibodies (1:50 dilution, Santa Cruz; 1:100 dilution, Upstate) and anti-p53 antibody (1:100 dilution, Santa Cruz) overnight at $4{ }^{\circ} \mathrm{C}$, and then incubated with HRP-conjugated biotinylated IgG. The sections were then visualized by DAB solution and counterstained with hematoxylin.

The immunohistochemically stained sections were examined by two independent pathologists who were blinded to the clinicopathological information. Each case was evaluated for the intensity of cell staining and the percentage of positive staining cells. The intensity of the cell staining was graded according to the following scale: 0 (negative), 1 (weak), 2 (moderate) and 3 (strong). The extent of staining was evaluated by the percentage of positive cells: 0 (negative), 1 (1-25\%), 2 (26-50\%), 3 (51-75\%) and 4 (76-100\%). The final score of each sample was assessed by the results of the intensity and extent of staining. Therefore, the expression of SIRT1 was defined as follows: 0 (negative, -), 1-4 (low expression, +), 5-8 (moderate expression, ++ ) and 9-12 (high expression, +++ ).

\section{RNA Isolation and $q R T-P C R$}

Total RNA was isolated from tissues with TRIzol reagent (Invitrogen) according to the manufacturer's instructions. cDNA was synthesized with RevertAid ${ }^{\text {TM }}$ First Strand cDNA Synthesis Kit (Thermo Fisher Scientific). qRT-PCR was performed with SYBR Green Master Mix (TaKaRa) according to the manufacturer's protocols. The primers for SIRT1 and GAPDH were designed as follows: SIRT1: forward $5^{\prime}$-TAGGCGGCTTGATGGTAAT-3', reverse 5'-ATGGGTTCTTCTAAACTTGGACT-3'; GAPDH: forward 5'-CCACCCATGGCAAATTCC-3', reverse $5^{\prime}$-TGGGATTTCCATTGATGACAAG-3'.

\section{Cell Culture and Treatment}

GES and SGC 7901 cells were routinely cultured in RPMI-1640 medium (GIBCO) supplemented with $10 \%$ fetal bovine serum (GIBCO), $100 \mathrm{U} / \mathrm{ml}$ of penicillin sodium and $100 \mathrm{mg} / \mathrm{ml}$ streptomycin. Cells were either infected with lentiviral particles or subjected to one of the following treatments for further experiments: (1) 200 nM resveratrol [24] (Sigma) or (2) $10 \mu \mathrm{M}$ EX-527 [24] (Tocris).

\section{Inhibition of SIRT1 by RNA Interference}

A double-stranded oligonucleotide (5'-CCGGGAAGTTGACCTCCTCATTGTTTCAAGAGAACAATGAGGAGGTCAACTTCTTTTTG; SIRT1-targeting sequence underlined [25]) was cloned into the pGCSIL-GFP vector (GeneChem). The plasmid was packaged into lentiviral particles (LV-SIRT1-shRNA) by transfection of the packaging cell line 293T. A virus expressing a scrambled shRNA (LV-shRNA-Control) was used as a control. GES and SGC 7901 cells were infected with LV-SIRT1-shRNA or LV-shRNA-Control, and the purity of stable transfectants was evaluated by detecting green fluorescent protein (GFP) expression using an inverted fluorescence microscope.

\section{Expression of SIRT1 by the Lentiviral Vector System}

The coding sequence of SIRT1 (NM_012238) was amplified by reverse transcription PCR (RT-PCR). The primer sequences were designed as follows: forward, 5'-ATCGGGATCCCGCCACCATGGCGGACGAGGCGGCCCTC-3'; reverse, 5'-CGGGTACCGGTACTGATTTGTTTGATGGATAGTTCATG-3'. The PCR fragments and the GV166 plasmid (GeneChem) were digested with AgeI and BamHI and then ligated with T4 DNA ligase to produce the GV166-SIRT1 plasmid. The lentivirus-expressing SIRT1 (LV-SIRT1) and the empty control lentivirus (LV-Control) were produced in HEK-293T cells by cotransfection of the GV166-SIRT1 or the GV166 
plasmid and the packaging plasmids pHelper1.0 and pHelper2.0. GES and SGC 7901 cells were infected with LV-SIRT1 or LV-Control and screened with $2 \mu \mathrm{g} / \mathrm{ml}$ puromycin for 10 days. Drug-resistant colonies were pooled for further experiments.

\section{Western Blotting Analysis}

Cellular proteins were extracted and separated in SDS-PAGE gels. Proteins were then transferred semi-dry to a nitrocellulose membrane and non-specific binding was blocked with TBS containing 5\% non-fat dry milk for $2 \mathrm{~h}$ at room temperature. The membranes were incubated overnight at $4{ }^{\circ} \mathrm{C}$ with the following antibodies at the following dilutions: SIRT1 (1:1,000, Santa Cruz or Upstate), cyclin D1 (1:800, Santa Cruz), Cdk4 (1:800, Santa Cruz), Rb (1:1,000, Santa Cruz), Bax (1:1,000, Santa Cruz), Bcl-2 (1:1,000, Santa Cruz) and $\beta$-actin (1:2,000, Santa Cruz). After incubation with an appropriate peroxidase-conjugated secondary antibody, the reactive bands were developed using enhanced chemiluminescence solutions (Pierce).

\section{MTT Assay}

The stable infected GES and SGC 7901 cells were prepared as a single cell suspension and seeded into 96-well plates at a density of $2 \times 10^{3}$ cells/well ( $200 \mu$ f for each well). After 1, 2, 3, 4, 5, 6 or 7 days of culture, $20 \mu \mathrm{l}$ MTT solution ( $5 \mathrm{mg} / \mathrm{ml}$, Sigma-Aldrich) was added to each well followed by $4 \mathrm{~h}$ incubation. The supernatant was carefully removed, and $150 \mu \mathrm{l}$ of DMSO was used to dissolve the resultant formazan crystals. The absorbance values were detected with an ELISA reader (Bio-Rad Laboratories) at $490 \mathrm{~nm}$.

\section{Cell Cycle Analysis}

After serum deprivation for $24 \mathrm{~h}$, stable infected GES and SGC 7901 cells were routinely cultured for $48 \mathrm{~h}$. In addition, GES and SGC 7901 cells were treated with $10 \mu \mathrm{M}$ EX-527, $200 \mathrm{nM}$ resveratrol or DMSO for $48 \mathrm{~h}$. The treated cells were harvested and fixed in $70 \%$ ethanol. After treatment with RNase at $37^{\circ} \mathrm{C}$ for $30 \mathrm{~min}$, the cells were stained with propidium iodide $(10 \mu \mathrm{g} / \mathrm{ml})$ and the percentages of cells in different cell cycle phases were measured by a flow cytometer (Becton-Dickinson).

\section{Cell Apoptosis Analysis}

To measure cell apoptosis, the Cy 5.5/PE or the Cy 5.5/FITC staining method (Invitrogen) was applied. Serum deprivation was used to induce apoptosis in stable infected GES and SGC 7901 cells. In addition, GES and SGC 7901 cells were treated with $10 \mu \mathrm{M}$ EX-527, $200 \mathrm{nM}$ resveratrol or DMSO for $48 \mathrm{~h}$, followed by serum deprivation to induce apoptosis. Cells were washed twice with cold PBS and resuspended in binding buffer. The samples were then supplemented with Cy 5.5/PE or Cy 5.5/FITC and incubated for 15 min at room temperature. The proportion of the cell populations in early and late apoptosis was determined by fluorescence-activated cell sorting (FACS) analysis.

\section{Tumorigenicity in Nude Mice and in vivo Bioluminescence Imaging}

The nude mice were handled using the best humane practices in accordance with the NIH Animal Care Institutional Guidelines. The logarithmically growing cells were harvested and resuspended in PBS. Four groups of 4-week-old female BALB/c nu/nu mice (each group included five mice) were injected subcutaneously with $1 \times 10^{7}$ stable infected SGC 7901 cells or control cells. The mice were then monitored for overall health. After a 4-week observation period, bioluminescence imaging was used to analyze in vivo tumor formation. Bioluminescence was detected by the IVIS Imaging System (Xenogen) and the luciferase signals were automatically calculated by the IVIS Living Image (Xenogen) software. Finally, animals were sacrificed and tumors were weighed.

\section{Statistical Analysis}

Statistical analysis was performed using the SPSS version 17.0 software. $\chi^{2}$ test or Student's t test was used to determine differences in expression of SIRT1 between gastric cancer tissue and non-tumor tissues. The Kruskal-Wallis H and Mann-Whitney U tests were used to compare the differences of SIRT1 expression with various clinical pathological parameters. One-way ANOVA analysis or Student's t test was used to compare the differences in cell proliferation, cell cycle, cell apoptosis and tumorigenicity in nude mice. A $\mathrm{p}$ value $<0.05$ was considered statistically significant. 


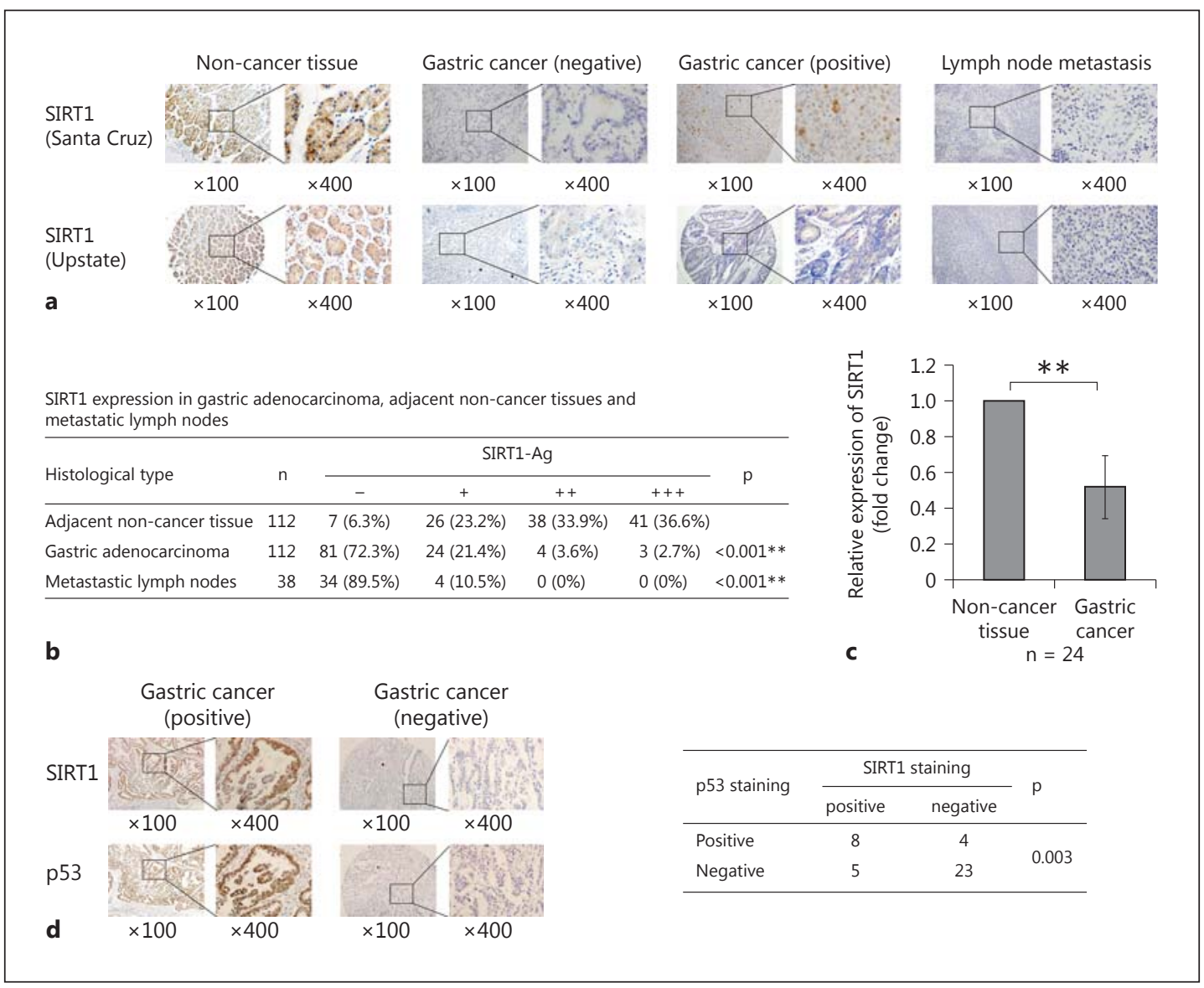

Fig. 1. SIRT1 expression was significantly reduced in gastric adenocarcinoma and correlated with p53 expression. a Two different SIRT1 antibodies were used for the immunohistochemical examination of SIRT1 expression in gastric adenocarcinoma, adjacent non-cancer tissues and in metastatic lymph nodes. Similar staining patterns were observed. b Statistical analysis revealed that SIRT1 expression was significantly reduced in gastric adenocarcinoma and metastatic lymph nodes. c qRT-PCR analysis of SIRT1 in gastric adenocarcinoma and paired adjacent non-cancer tissues. SIRT1 mRNA expression was decreased in gastric adenocarcinoma tissues. d p53 expression was detected by immunohistochemistry. The immunohistochemical results showed a significant association between SIRT1 expression and p53 expression in gastric adenocarcinoma. ${ }^{* *} \mathrm{p}<0.01$.

\section{Results}

SIRT1 Expression Was Significantly Reduced in Gastric Adenocarcinoma and Correlated with p53 Expression

Immunohistochemistry was performed to assess the expression of SIRT1 in 112 cases of primary gastric adenocarcinoma and corresponding adjacent non-tumor tissues, as well as in 38 cases of metastatic lymph nodes. To increase the reliability of our results, we applied two different SIRT1 antibodies (Santa Cruz and Upstate) for immunohistochemistry, both of which produced similar staining patterns. As shown in figure 1a and b, SIRT1 was detected in $93.7 \%$ of the adjacent non-tumor tissues, exhibiting moderate to intense staining which was mainly cytoplasmic. However, positive nuclear and/or cytoplasmic staining was detected in $27.7 \%$ of the gastric adenocarcinoma tissues and in $10.5 \%$ of the metastatic lymph nodes. There was a positive correlation between SIRT1 expression and 
Table 1. Correlation of SIRT1 expression with clinicopathological features

\begin{tabular}{|c|c|c|c|c|c|c|c|}
\hline \multicolumn{2}{|c|}{ Clinicopathological features } & \multirow[t]{2}{*}{$\mathrm{n}$} & \multicolumn{4}{|c|}{ SIRT1 } & \multirow[t]{2}{*}{$\mathrm{p}$} \\
\hline & & & - & + & ++ & +++ & \\
\hline \multirow[t]{2}{*}{ Age, years } & $\geq 60$ & 48 & 36 & 6 & 3 & 3 & \multirow{2}{*}{0.918} \\
\hline & $<60$ & 64 & 46 & 15 & 1 & 2 & \\
\hline \multirow[t]{2}{*}{ Sex } & M & 83 & 58 & 17 & 4 & 4 & \multirow{2}{*}{0.169} \\
\hline & $\mathrm{F}$ & 29 & 24 & 4 & 0 & 1 & \\
\hline \multirow[t]{2}{*}{ TNM stage } & I and II & 77 & 58 & 14 & 2 & 3 & \multirow{2}{*}{0.416} \\
\hline & III and IV & 35 & 24 & 7 & 2 & 2 & \\
\hline \multirow[t]{3}{*}{ Histological grade } & well-differentiated & 9 & 8 & 0 & 1 & 0 & \multirow{3}{*}{$0.026^{*}$} \\
\hline & moderately differentiated & 35 & 19 & 14 & 1 & 1 & \\
\hline & poorly differentiated & 68 & 55 & 7 & 2 & 4 & \\
\hline \multirow{2}{*}{$\begin{array}{l}\text { Lymph node } \\
\text { metastasis }\end{array}$} & absence & 49 & 33 & 9 & 4 & 3 & \multirow{2}{*}{0.151} \\
\hline & presence & 63 & 49 & 12 & 0 & 2 & \\
\hline \multirow[t]{2}{*}{ Distant metastasis } & absence & 112 & 82 & 21 & 4 & 5 & \multirow{2}{*}{ n.s. } \\
\hline & presence & 0 & 0 & 0 & 0 & 0 & \\
\hline
\end{tabular}

$* \mathrm{p}<0.05$. n.s. $=$ Not significant.

histological tumor type, but no significant correlation between SIRT1 expression and sex, age, TNM stage or metastasis, which might be attributed to the low expression of SIRT1 in gastric adenocarcinoma (table 1).

Moreover, SIRT1 mRNA expression was evaluated in 24 cases of gastric adenocarcinoma and corresponding non-tumor mucosa tissues by qRT-PCR analysis. Consistent with our immunohistochemical results, qRT-PCR analysis revealed that the SIRT1 mRNA expression levels were significantly lower in gastric cancer tissues compared with the corresponding non-tumor mucosa tissues (fig. 1c).

p53 is an important non-histone deacetylation target for SIRT1. SIRT1 has been shown to deacetylate p53, thereby repressing p53-dependent cell cycle arrest and apoptosis in response to DNA damage and oxidative stress $[5,13,26]$. Thus we evaluated the expression of p53 in our tissue samples. We found that p53 expression was detected in $30 \%$ of gastric adenocarcinoma tissue samples. Immunohistochemical analysis revealed a significant correlation between the expression of SIRT1 and p53 (p = 0.003) (fig. 1d). As we know, wild-type p53 protein, rapidly removed from the nucleus, is usually not detectable due to its short halflife. However, mutant p53 becomes immunohistochemically detectable because of its prolonged half-life, which results in inefficient DNA repair and the emergence of genetically unstable cells [27]. We hypothesized that the expression of SIRT1 in gastric adenocarcinoma might compensate for the nuclear accumulation of mutant p53 protein.

\section{SIRT1 Inhibited Cell Growth and Proliferation in vitro}

To further explore the effects of SIRT1 on the development and progression of gastric cancer, we infected GES and SGC 7901 cells with the following lentiviruses: LV-shRNA-Control, LV-SIRT1-shRNA, LV-Control or LV-SIRT1. Western blotting confirmed a marked reduction of SIRT1 by LV-SIRT1-shRNA infection and a significant overexpression of SIRT1 by LV-SIRT1 infection (fig. 2a). As indicated by the MTT assay in figure 2b, LV-SIRT1-shRNA-infected cells exhibited a significantly increased rate of cell proliferation compared with LV-shRNA-Control- 


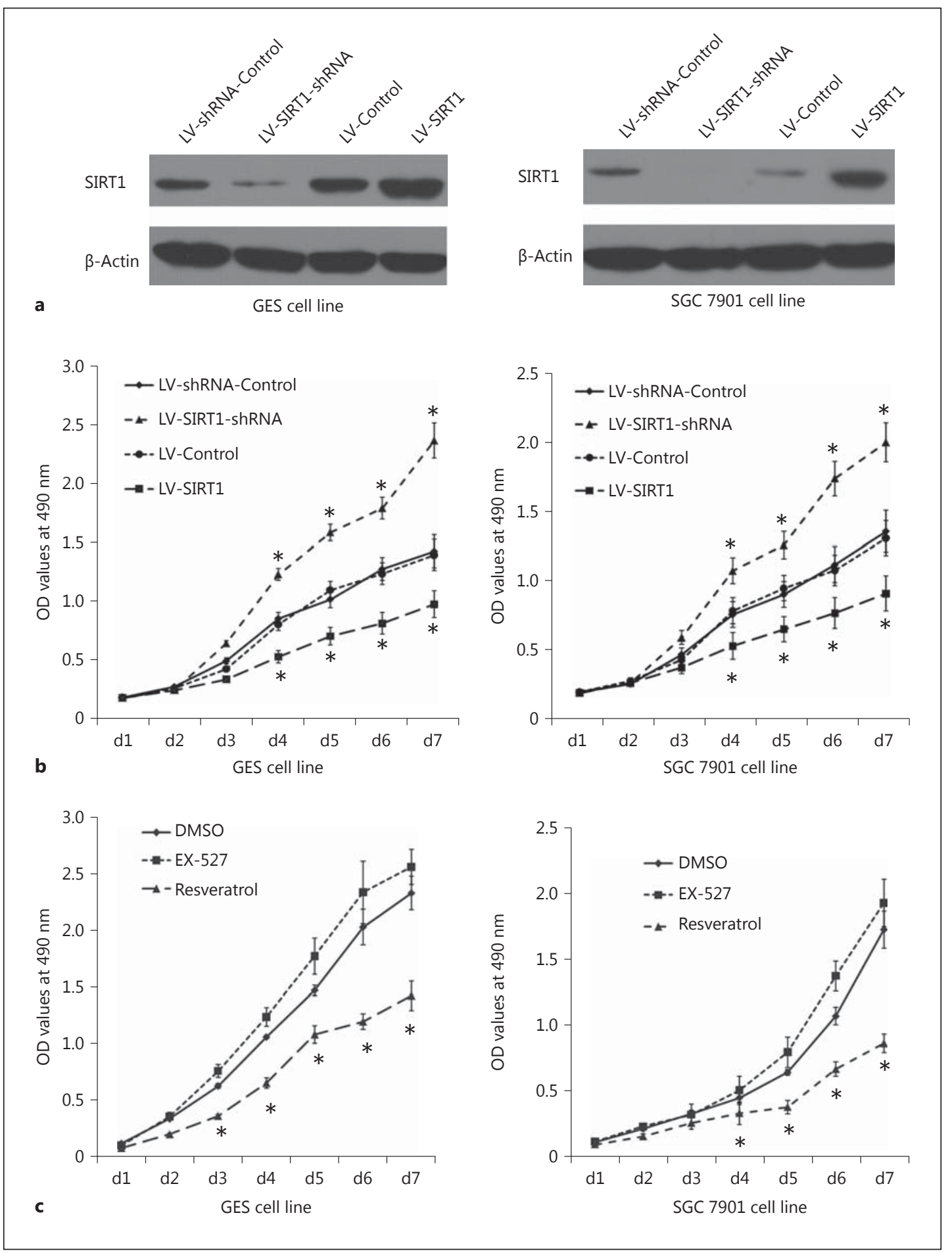

Fig. 2. SIRT1 inhibited the proliferation of GES and SGC 7901 cells in vitro. a The validation of LV-SIRT1shRNA or LV-SIRT1 infection was confirmed by Western blotting analysis. $\mathbf{b}$ The MTT assay was performed using stable infected GES and SGC 7901 cells. Cell numbers were evaluated by absorbance at $490 \mathrm{~nm}$. The inhibition of SIRT1 increased the proliferation rate of GES and SGC 7901 cells, whereas the ectopic overexpression of SIRT1 suppressed their proliferation. c The MTT assay was performed using GES and SGC 7901 cells treated with $10 \mu \mathrm{M}$ EX-527 or $200 \mathrm{nM}$ resveratrol. Cell numbers were evaluated by absorbance at $490 \mathrm{~nm}$. EX-527 promoted the growth of both GES and SGC 7901 cells, whereas the activation of SIRT1 by resveratrol inhibited cells proliferation. $* \mathrm{p}<0.05$. 
Zhang et al.: SIRT1 Is Reduced in Gastric Adenocarcinoma and Acts as a Potential Tumor Suppressor in Gastric Cancer

infected cells, whereas overexpression of SIRT1 by LV-SIRT1 strongly inhibited cell proliferation in GES and SGC 7901 cells. To further evaluate the role of endogenous SIRT1 in regulating cell growth, $10 \mu \mathrm{M}$ EX-527 (SIRT1 inhibitor) or $200 \mathrm{~nm}$ resveratrol (SIRT1 activator) was administered to change the activity of SIRT1. As shown in figure 2c, $10 \mu \mathrm{M}$ EX-527 promoted cell growth in both GES and SGC 7901 cells. However, when treated with $200 \mathrm{nM}$ resveratrol, the proliferation rates of GES and SGC 7901 cells were significantly lower than those of control-treated cells.

\section{SIRT1 Arrested Cell Cycle Progression by Regulating the Expression of Cell Cycle-Related} Proteins

Sustaining proliferation is an important hallmark of cancer. Thus, we performed flow cytometric analysis to assess the effects of SIRT1 on cell cycle. As shown in figure 3a and b, after serum deprivation for $24 \mathrm{~h}$, the proportion of cells in G1 phase significantly decreased in LV-SIRT1-shRNA-infected cells ( $58.5 \pm 0.8 \%$ vs. $41.9 \pm 0.6 \%$ for GES cells and $46.5 \pm 0.7 \%$ vs. $37.8 \pm 0.6 \%$ for SGC 7901 cells, $\mathrm{p}<0.05$ ) compared with LV-shRNA-Control-infected cells. In contrast, the proportion of cells in G1 phase significantly increased in LV-SIRT1-infected cells $(57.6 \pm 1.0 \%$ vs. $64.6 \pm 1.3 \%$ for GES cells and $45.1 \pm 1.2 \%$ vs. $58.9 \pm 1.6 \%$ for SGC 7901 cells, $\mathrm{p}<0.05$ ). Furthermore, cell cycle analysis was performed on GES and SGC 7901 cells treated with $10 \mu \mathrm{M}$ EX-527 or $200 \mathrm{~nm}$ resveratrol. Compared with control cells, EX-527 caused a significant reduction of the proportion of cells in G1 phase $(59.8 \pm 0.9 \%$ vs. $47.4 \pm 0.8 \%$ for GES cells and $55.3 \pm 1.2 \%$ vs. $41.1 \pm 1.0 \%$ for SGC 7901 cells, $p<0.05$ ). In contrast, resveratrol caused a significant increase in the proportion of cells in G1 phase (59.8 $\pm 0.9 \%$ vs. $69.6 \pm$ $1.1 \%$ for GES cells and $55.3 \pm 1.2 \%$ vs. $68.1 \pm 1.6 \%$ for SGC 7901 cells, $p<0.05$ ) (fig. 3 c, d). These results indicated that inhibition of cell proliferation by SIRT1 occurred partially through arrest of cell cycle progression.

To confirm the cell cycle arrest caused by SIRT1, we examined the expression of cell cycle-related proteins, including two key cell cycle regulators, cyclin D1 and Cdk4. Cyclin D1 binds to Cdk4, forming the active cyclin D1-Cdk4 complex, which pushes the cell from G1 to S phase $[28,29]$. Western blotting indicated that knockdown of SIRT1 resulted in increased expression levels of cyclin D1 and Cdk4 without affecting the expression of Rb. However, overexpression of SIRT1 induced by LV-SIRT1 reduced the expression levels of cyclin D1 and Cdk4 (fig. 3e, f).

\section{SIRT1 Induced Cell Apoptosis by Targeting Cell Apoptosis-Related Proteins}

Resistance to cell death is another important hallmark of cancer, and apoptosis serves as a natural barrier to cancer development. Here, we analyzed the effect of SIRT1 on cell apoptosis. Knockdown of SIRT1 decreased the apoptotic index of both cells lines $(7.6 \pm 0.7 \%$ vs. $3.8 \pm 0.6 \%$ for GES cells and $9.8 \pm 0.8 \%$ vs. $4.8 \pm 0.5 \%$ for SGC 7901 cells, $p<0.05$ ), whereas SIRT1 overexpression significantly increased the percentage of apoptotic cells ( $6.3 \pm 0.5 \%$ vs. $16.5 \pm 1.0 \%$ for GES cells and $9.6 \pm 0.8 \%$ vs. $21.6 \pm 2.1 \%$ for SGC 7901 cells, $\mathrm{p}<0.05$ ) (fig. $4 \mathrm{a}$, b). Moreover, when we applied EX-527 or resveratrol to GES and SGC 7901 cells, we observed that the inhibition of SIRT1 by EX-527 suppressed cell apoptosis in the GES cell line, whereas the activation of SIRT1 by resveratrol promoted cell apoptosis in GES and SGC 7901 cells (fig. 4c, d).

To further confirm the role of SIRT1 in cell apoptosis, we analyzed the protein expression of apoptosis-associated factors. Compared with LV-shRNA-Control-infected cells, LV-SIRT1shRNA-infected cells exhibited significantly increased Bcl-2 and decreased Bax expression levels. Conversely, a significant increase in Bax expression and a decrease in Bcl-2 expression levels were observed after LV-SIRT1 infection (fig. 4e, f). 

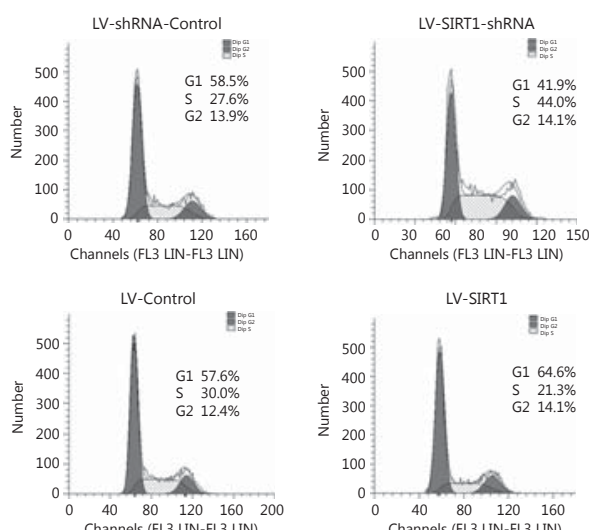

GES cell line
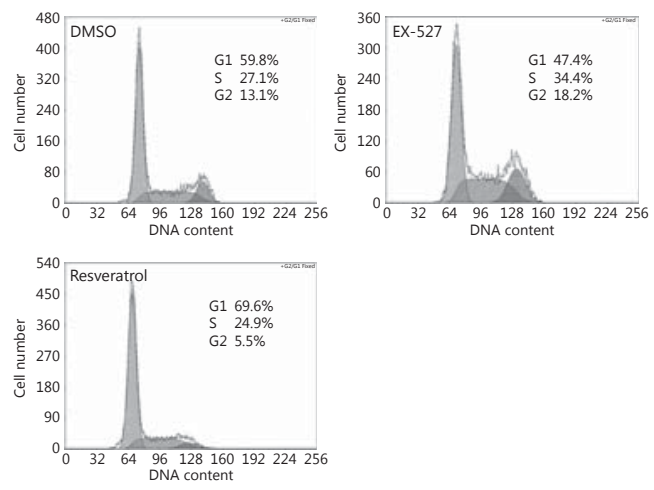

c

GES cell line

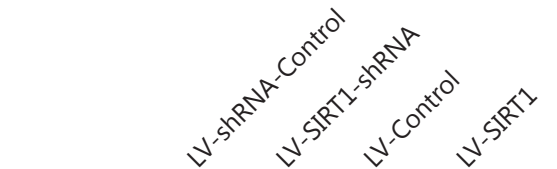

Cyclin D1

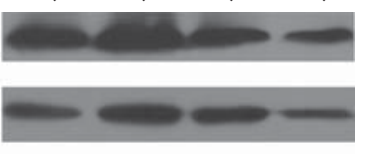

Cdk4

$\mathrm{Rb}$

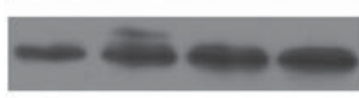

$\beta$-Actin

e

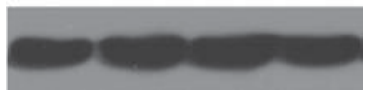

GES cell line
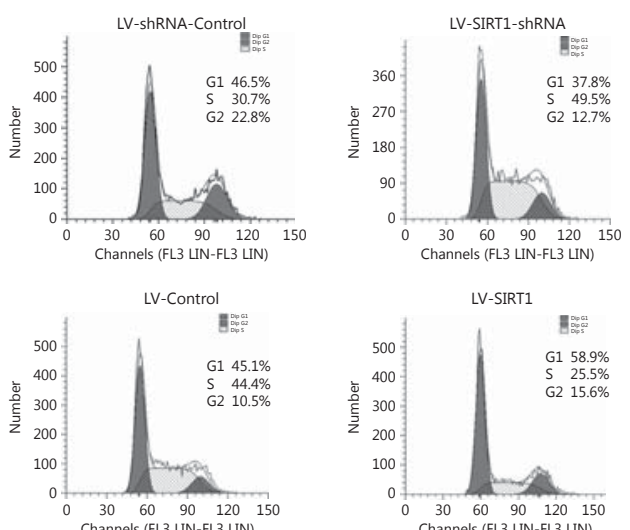

b

SGC 7901 cell line
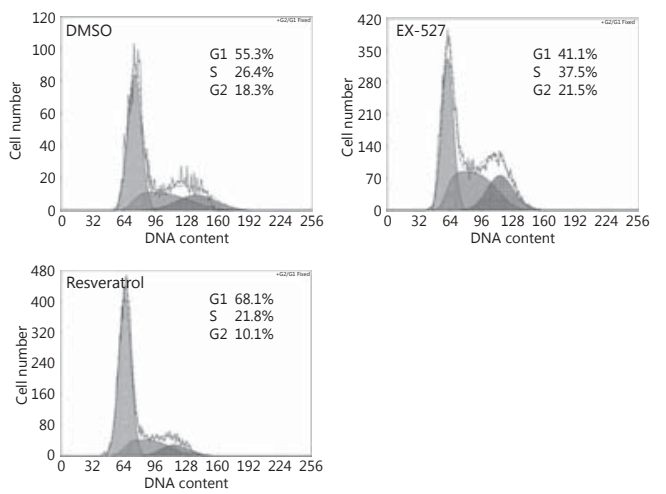

d

SGC 7901 cell line

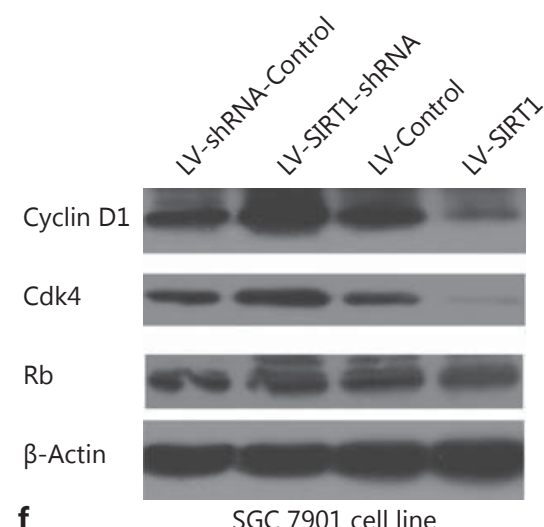

SGC 7901 cell line

Fig. 3. SIRT1 arrested cell cycle progression in GES and SGC 7901 cells. a, b Cell cycle analysis of GES and SGC 7901 stable cells expressing either LV-SIRT1-shRNA or LV-SIRT1 was performed by flow cytometric analysis, and the percentage of cells in each cell cycle phase was indicated. The G1-S phase transition was accelerated by knockdown of SIRT1 but was inhibited by SIRT1 overexpression. c, d GES and SGC 7901 cells were treated with $10 \mu \mathrm{M}$ EX-527 or $200 \mathrm{~nm}$ resveratrol for $48 \mathrm{~h}$. Cell cycle analysis was performed to analyze the percentage of cells in each cell cycle phase. EX-527 caused a decreased percentage of the G1 phase, whereas resveratrol caused a G1-S phase arrest. e, f Expression of cell cycle-regulating proteins (cyclin D1, Cdk4 and $\mathrm{Rb}$ ) was assessed by Western blotting analysis. Ectopic overexpression of SIRT1 reduced the expression levels of cyclin D1 and Cdk4. 


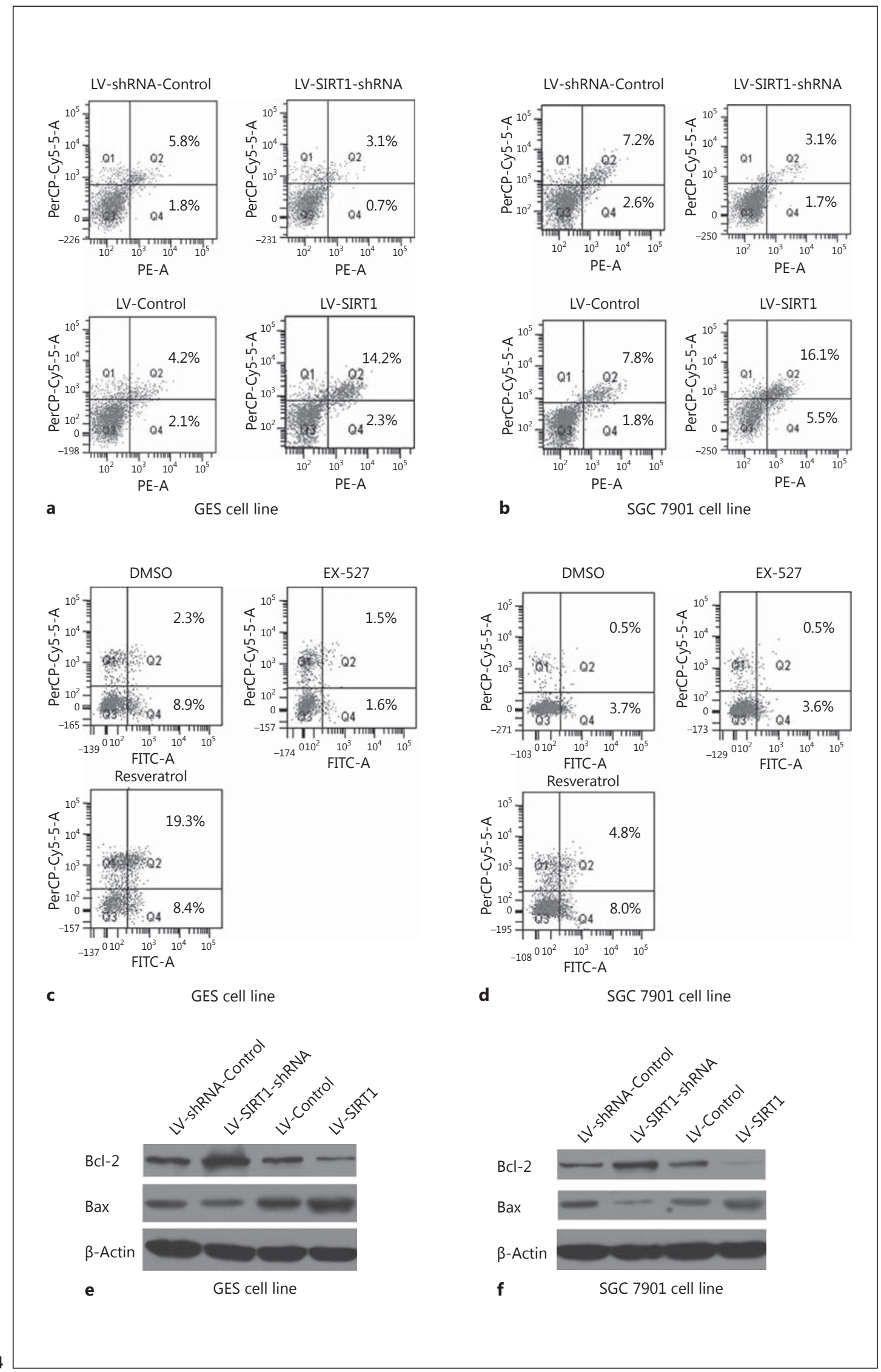




\section{SIRT1 Inhibited Tumor Formation in Nude Mice}

We further investigated the effects of SIRT1 on tumorigenesis in vivo. The tumor formation potential of stable infected SGC 7901 cells was assessed in xenograft animals. The stable infected SGC 7901 cells were inoculated subcutaneously into female nude mice. As shown in figure 5, SIRT1 knockdown cells exhibited higher luciferase intensities and tumor volumes than control-treated cells. Conversely, overexpression of SIRT1 dramatically inhibited tumor formation in vivo, and the average luciferase signals and tumor weights were lower for LV-SIRT1-infected cells than for LV-Control-infected cells.

\section{Discussion}

Our study was the first to report that the expression of SIRT1 is reduced in gastric adenocarcinoma. We demonstrated that the repression of SIRT1 increases gastric cancer cell proliferation in vitro and tumorigenesis in vivo. In contrast, the overexpression or activation of SIRT1 suppresses gastric cancer cell growth and tumor formation. Our findings identify SIRT1 as a potential tumor suppressor in gastric cancer.

SIRT1 is a multifaceted, $\mathrm{NAD}^{+}$-dependent protein deacetylase that is involved in a wide variety of cellular processes [6]. Several studies have found a significant upregulation of SIRT1 in human prostate cancer [8], gastric cancer [9], colon cancer [10], breast cancer [11], hepatocellular carcinoma [12] and non-melanoma skin cancer [30]. SIRT1 deacetylates and inhibits the activities of p53 [13], FOXO transcription factor family member [14], Ku70 [31] and E2F1 [15], which may block cell apoptosis and promote cell survival in response to DNA damage and oxidative stress. The overexpression of SIRT1 has also been shown to repress the expression or activity of tumor suppressor and DNA repair genes, including WRN [32], Rb [16], MLH1 [33] and NBS1 [34]. Inhibition of SIRT1 by small molecule inhibitors or downregulation of SIRT1 by siRNA results in not only cell growth arrest and apoptosis [35, 36], but also in reactivation of tumor suppressor genes without loss of promoter DNA hypermethylation [33]. Furthermore, several tumor suppressors, including HIC1 [37] and DBC1 [38], have been reported to repress the expression and activity of SIRT1. However, some studies have also found a reduction of SIRT1 expression in breast cancer [17], hepatic carcinoma [18], colon cancer [19], prostate carcinoma [18], glioblastoma [18] and bladder carcinoma [18]. SIRT1 deacetylates and inhibits the activities of nuclear factor $\kappa B$ [39], $\beta$-catenin [20], survivin [17] and c-Myc [40], which leads to suppress tumor formation and growth. The activation of SIRT1 by resveratrol inhibits proliferation and promotes apoptosis in several cancer cells [41, 42]. Furthermore, studies using SIRT1 transgenic mice strongly support the hypothesis that SIRT1 is a tumor suppressor. Stunkel et al. [10] demonstrated that SIRT1 overexpression inhibited the growth of colon cancer cells in the $\mathrm{APC}^{-/+}$mouse model. Another study by Wang et al. [18] suggested that SIRT1 ${ }^{+/-}$p53 $3^{+/-}$mice developed spontaneous tumors in multiple tissues,

Fig. 4. SIRT1-induced cell apoptosis in GES and SGC 7901 cells. a, b After Cy 5.5 and PE staining, the apoptosis cells of GES and SGC 7901 cells infected with LV-SIRT1-shRNA or LV-SIRT1 were measured by FACS. Knockdown of SIRT1 decreased the apoptotic index of GES and SGC 7901 cells, whereas SIRT1 overexpression increased the percentage of apoptotic cells. c, d GES and SGC 7901 cells were treated with $10 \mu \mathrm{M} \mathrm{EX-527} \mathrm{or}$ $200 \mathrm{~nm}$ resveratrol for $48 \mathrm{~h}$, and the percentage of apoptotic cells was measured by flow cytometry analysis after FITC and Cy 5.5 staining. EX-527 suppressed cell apoptosis, whereas resveratrol promoted cell apoptosis. e, f Apoptotic markers Bax and Bcl-2 were detected by Western blotting analysis. Overexpression of SIRT1 conferred by LV-SIRT1 caused a significant increase in Bax expression and a reduction in Bcl-2 expression. 


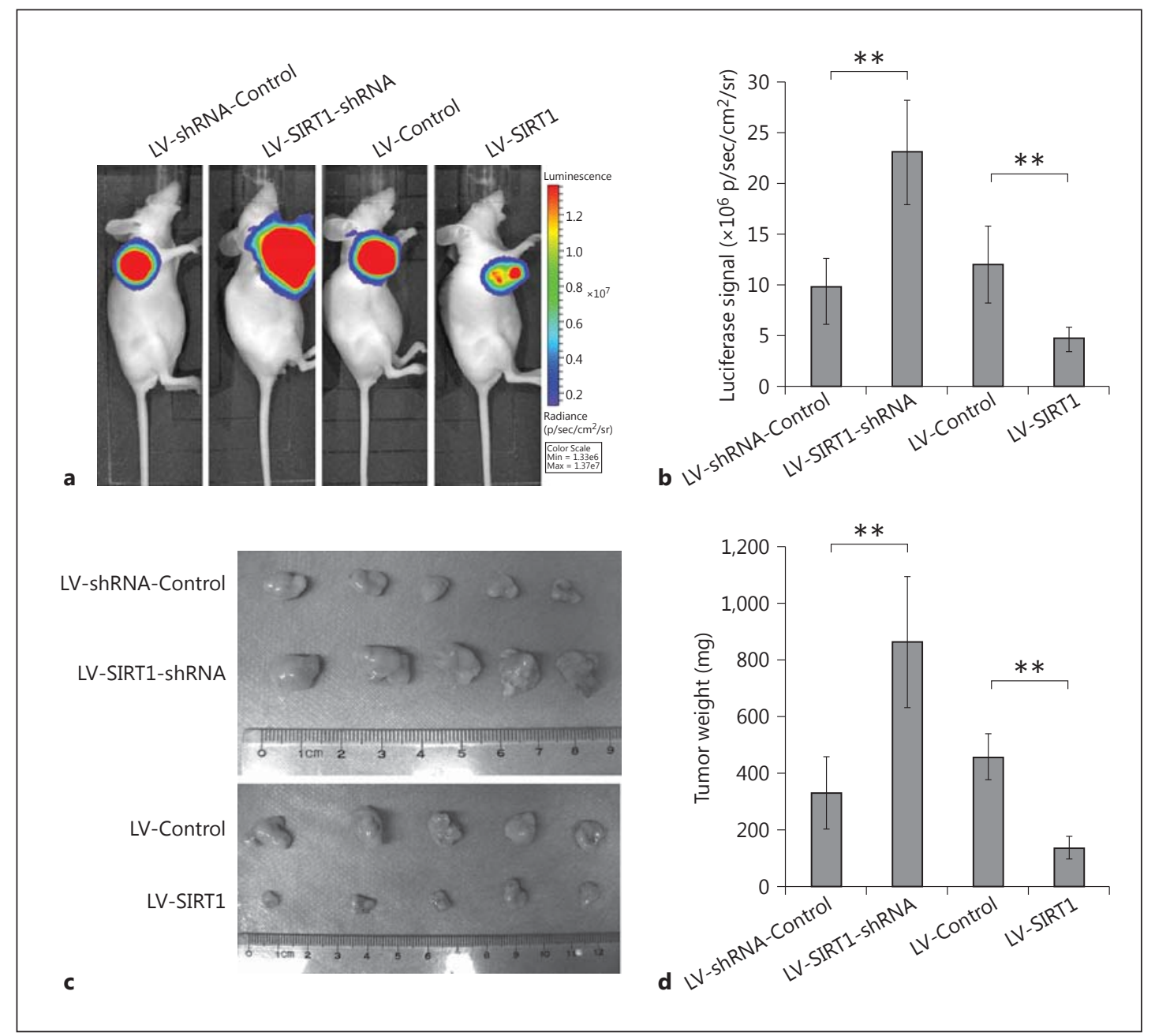

Fig. 5. SIRT1 inhibited tumor formation in nude mice. a In vivo bioluminescence imaging was used to detect tumor formation by an IVIS Imaging System. The representative graphs were shown. $\mathbf{b}$ The luciferase signals were automatically calculated by the IVIS Living Image software. The average luciferase signals in different groups were calculated and compared. c Nude mice were sacrificed and tumor formation was shown. The overexpression of SIRT1 dramatically inhibited tumor formation. $\mathbf{d}$ The average tumor weight was measured and quantified. ${ }^{* *} \mathrm{p}<0.01$.

whereas activation of SIRT1 reduced tumorigenesis. Herranz et al. [21] found that transgenic mice moderately overexpressing SIRT1 presented lower levels of DNA damage, fewer spontaneous carcinomas and sarcomas. All of these conflicting findings imply that SIRT1 might function as a promoter or suppressor of tumorigenesis, depending on the cellular context, type and stage of the tumor.

With regard to gastric cancer, Cha et al. [9] found that SIRT1 was expressed in a high proportion of gastric carcinomas and that the expression of SIRT1 was significantly associated with clinicopathological variables such as tumor stage, lymph node metastasis, tumor invasion and histological tumor types, indicating that SIRT1 would play an oncogene-like role in tumorigenesis. They further concluded that SIRT1 expression was significantly associated with shorter overall and relapse-free survival. In order to gain a more solid conclusion, we used two different SIRT1 antibodies, including the same one used by Cha et al. [9], to evaluate paired gastric adenocarcinoma specimens. Unexpectedly, we found that SIRT1 expression 
was reduced in gastric carcinoma compared with the adjacent non-tumor tissues. Accordingly we also found that the SIRT1 mRNA expression level was significantly lower in gastric cancer tissues. However, we found no significant correlation between the expression of SIRT1 and sex, age, TNM stage or metastasis. Several possibilities might account for this discrepancy. One possible explanation might be that we analyzed a different type of gastric cancer. The diffuse and mixed types of gastric cancer were mainly examined in the study by Cha et al., whereas we focused on intestinal type in our study. The intestinal type is the most common type of gastric cancer in high-prevalence areas such as China [43]. Another possible explanation is that the tumors were obtained from patients of different ethnic backgrounds. Moreover, the number of samples analyzed in Cha et al.'s study may not have been sufficient to evaluate the SIRT1 expression in non-cancer gastric tissue (only 10 samples were stained for SIRT1) [9].

Also, Cha et al. did not explore the potential critical roles of SIRT1. Our in vitro or in vivo data strongly suggested that reduced expression of SIRT1 promoted the development and tumorigenesis of gastric cancer. We found that both overexpression of SIRT1 and activation of endogenous SIRT1 resulted in a significant inhibition of cell proliferation. We also found that overexpression of SIRT1 reduced tumor xenograft formation in nude mice. In contrast, inhibition of SIRT1 function by RNA interference or EX-527 treatment promoted cellular proliferation in vivo and in vitro. Although the underlying mechanism by which SIRT1 inhibits cell proliferation remains unclear, our study suggests that its effects are mediated at least partially through regulation of cell cycle progression. We found that the G1-S phase transition was inhibited by the upregulation of SIRT1 and accelerated by the downregulation of SIRT1. As cyclin D1, Cdk4 and Rb are key regulators of cell cycle progression [29], we examined the effects of SIRT1 on the expression levels of these key regulators. We found that the expression of cyclin D1 and Cdk4 was significantly decreased by the ectopic overexpression of SIRT1. Consistently, knockdown of SIRT1 significantly increased cyclin D1 and $\mathrm{Cdk} 4$ expression levels, without affecting the expression of $\mathrm{Rb}$. These results suggest that the contribution of SIRT1 to cell cycle arrest might be through the regulation of cell cycle-related proteins.

Over the last two decades, researchers have established that apoptosis serves as a natural barrier to cancer development [44], and resisting apoptosis is one of the major characteristics that cancer cells undergo to survive and proliferate [17]. Tumor cells utilize a variety of strategies to limit or to escape apoptosis [44]. Several studies have revealed the effects of SIRT1 on cell apoptosis in a profile of cancer cell lines [35, 45-47], but there have been no reports regarding gastric cancer cells. The present study showed that overexpression of SIRT1 induced cell death, whereas loss of endogenous SIRT1 promoted cell survival. Moreover, altering the activity of endogenous SIRT1 by resveratrol or EX-527 produced similar results. These findings are consistent with previous studies demonstrating that resveratrol induces cell apoptosis through the activation of endogenous SIRT1 [42, 48, 49]. In further support of our findings, the expression levels of Bax and Bcl-2 (key regulators in regulation of pro- and anti-apoptotic processes [50]) were detected. We discovered that Bax was significantly increased upon upregulation of SIRT1 while a reduced expression of Bcl-2 was observed. Considering these results, we propose that SIRT1 might induce cell apoptosis by targeting cell apoptosis-related proteins.

In conclusion, our data suggest that the expression of SIRT1 is reduced in gastric adenocarcinoma. Our study demonstrates that repression of SIRT1 increases gastric cancer cell proliferation in vitro and tumorigenesis in vivo. In contrast, overexpression or activation of SIRT1 suppresses gastric cancer cell growth and tumor formation. Therefore, these findings identify SIRT1 as a potential tumor suppressor in gastric cancer and provide a rationale for using SIRT1 activators as therapeutic agents. 


\section{Acknowledgements}

This work was supported by the National Natural Science Foundation of China (81430072, 81120108005, 81372609, 81421003, 81272652, 81225003, 81227901, 81370504), Project from Ministry of Science and Technology of China (2015BAI13B07, 2012AA02A504, 2012AA02A203-A03, 2012ZX09303011) as well as Promoting Project of Xijing Hospital XJZT13G01.

\section{Disclosure Statement}

No potential conflicts of interest are disclosed.

\section{References}

1 Kamangar F, Dores GM, Anderson WF: Patterns of cancer incidence, mortality, and prevalence across five continents: defining priorities to reduce cancer disparities in different geographic regions of the world. J Clin Oncol 2006;24:2137-2150.

-2 Leung WK, Wu MS, Kakugawa Y, Kim JJ, Yeoh KG, Goh KL, et al: Screening for gastric cancer in Asia: current evidence and practice. Lancet Oncol 2008;9:279-287.

3 Blander G, Guarente L: The Sir2 family of protein deacetylases. Annu Rev Biochem 2004;73:417-435.

4 Voelter-Mahlknecht S, Mahlknecht U: Cloning, chromosomal characterization and mapping of the NADdependent histone deacetylases gene sirtuin 1. Int J Mol Med 2006;17:59-67.

5 Deng CX: SIRT1, is it a tumor promoter or tumor suppressor? Int J Biol Sci 2009;5:147-152.

6 Brooks CL, Gu W: How does SIRT1 affect metabolism, senescence and cancer? Nat Rev Cancer 2009;9:123128.

7 Finkel T, Deng CX, Mostoslavsky R: Recent progress in the biology and physiology of sirtuins. Nature 2009; 460:587-591.

8 Huffman DM, Grizzle WE, Bamman MM, Kim JS, Eltoum IA, Elgavish A, et al: SIRT1 is significantly elevated in mouse and human prostate cancer. Cancer Res 2007;67:6612-6618.

-9 Cha EJ, Noh SJ, Kwon KS, Kim CY, Park BH, Park HS, et al: Expression of DBC1 and SIRT1 is associated with poor prognosis of gastric carcinoma. Clin Cancer Res 2009;15:4453-4459.

10 Stunkel W, Peh BK, Tan YC, Nayagam VM, Wang X, Salto-Tellez M, et al: Function of the SIRT1 protein deacetylase in cancer. Biotechnol J 2007;2:1360-1368.

11 Lee H, Kim KR, Noh SJ, Park HS, Kwon KS, Park BH, et al: Expression of DBC1 and SIRT1 is associated with poor prognosis for breast carcinoma. Hum Pathol 2011;42:204-213.

-12 Chen J, Zhang B, Wong N, Lo AW, To KF, Chan AW, et al: Sirtuin 1 is upregulated in a subset of hepatocellular carcinomas where it is essential for telomere maintenance and tumor cell growth. Cancer Res 2011;71:41384149.

13 Vaziri H, Dessain SK, Ng Eaton E, Imai SI, Frye RA, Pandita TK, et al: hSIR2(SIRT1) functions as an NADdependent p53 deacetylase. Cell 2001;107:149-159.

14 Motta MC, Divecha N, Lemieux M, Kamel C, Chen D, Gu W, et al: Mammalian SIRT1 represses forkhead transcription factors. Cell 2004;116:551-563.

15 Wang C, Chen L, Hou X, Li Z, Kabra N, Ma Y, et al: Interactions between E2F1 and SirT1 regulate apoptotic response to DNA damage. Nat Cell Biol 2006;8:1025-1031.

16 Wong S, Weber JD: Deacetylation of the retinoblastoma tumour suppressor protein by SIRT1. Biochem J 2007; 407:451-460.

17 Wang RH, Zheng Y, Kim HS, Xu X, Cao L, Luhasen T, et al: Interplay among BRCA1, SIRT1, and Survivin during BRCA1-associated tumorigenesis. Mol Cell 2008;32:11-20.

18 Wang RH, Sengupta K, Li C, Kim HS, Cao L, Xiao C, et al: Impaired DNA damage response, genome instability, and tumorigenesis in SIRT1 mutant mice. Cancer Cell 2008;14:312-323.

19 Kabra N, Li Z, Chen L, Li B, Zhang X, Wang C, et al: SirT1 is an inhibitor of proliferation and tumor formation in colon cancer. J Biol Chem 2009;284:18210-18217.

20 Firestein R, Blander G, Michan S, Oberdoerffer P, Ogino S, Campbell J, et al: The SIRT1 deacetylase suppresses intestinal tumorigenesis and colon cancer growth. PLoS One 2008;3:e2020.

-21 Herranz D, Munoz-Martin M, Canamero M, Mulero F, Martinez-Pastor B, Fernandez-Capetillo 0, et al: Sirt1 improves healthy ageing and protects from metabolic syndrome-associated cancer. Nat Commun 2010;1:3.

-22 Sung JY, Kim R, Kim JE, Lee J: Balance between SIRT1 and DBC1 expression is lost in breast cancer. Cancer Sci 2010;101:1738-1744.

-23 Powell MJ, Casimiro MC, Cordon-Cardo C, He X, Yeow WS, Wang C, et al: Disruption of a Sirt1-dependent autophagy checkpoint in the prostate results in prostatic intraepithelial neoplasia lesion formation. Cancer Res 2011;71:964-975. 
Nie Y, Erion DM, Yuan Z, Dietrich M, Shulman GI, Horvath TL, et al: STAT3 inhibition of gluconeogenesis is downregulated by SirT1. Nat Cell Biol 2009;11:492-500.

-25 Cohen HY, Miller C, Bitterman KJ, Wall NR, Hekking B, Kessler B, et al: Calorie restriction promotes mammalian cell survival by inducing the SIRT1 deacetylase. Science 2004;305:390-392.

26 Luo J, Nikolaev AY, Imai S, Chen D, Su F, Shiloh A, et al: Negative control of p53 by Sir2alpha promotes cell survival under stress. Cell 2001;107:137-148.

-27 Soussi T: p53 antibodies in the sera of patients with various types of cancer: a review. Cancer Res 2000;60: 1777-1788.

28 Nigg EA: Cyclin-dependent protein kinases: key regulators of the eukaryotic cell cycle. Bioessays 1995; 17: 471-480.

29 Murray AW: Recycling the cell cycle: cyclins revisited. Cell 2004;116:221-234.

- 30 Hida Y, Kubo Y, Murao K, Arase S: Strong expression of a longevity-related protein, SIRT1, in Bowen’s disease. Arch Dermatol Res 2007;299:103-106.

-31 Jeong J, Juhn K, Lee H, Kim SH, Min BH, Lee KM, et al: SIRT1 promotes DNA repair activity and deacetylation of Ku70. Exp Mol Med 2007;39:8-13.

-32 Coleman RE, Nath AK, Schneider I, Song GH, Klein TA, Milhous WK: Prevention of sporogony of Plasmodium falciparum and P. berghei in Anopheles stephensi mosquitoes by transmission-blocking antimalarials. Am J Trop Med Hyg 1994;50:646-653.

-33 Pruitt K, Zinn RL, Ohm JE, McGarvey KM, Kang SH, Watkins DN, et al: Inhibition of SIRT1 reactivates silenced cancer genes without loss of promoter DNA hypermethylation. PLoS Genet 2006;2:e40.

-34 Yuan Z, Zhang X, Sengupta N, Lane WS, Seto E: SIRT1 regulates the function of the Nijmegen breakage syndrome protein. Mol Cell 2007;27:149-162.

-35 Peck B, Chen CY, Ho KK, Di Fruscia P, Myatt SS, Coombes RC, et al: SIRT inhibitors induce cell death and p53 acetylation through targeting both SIRT1 and SIRT2. Mol Cancer Ther 2010;9:844-855.

-36 Ota H, Tokunaga E, Chang K, Hikasa M, Iijima K, Eto M, et al: Sirt1 inhibitor, Sirtinol, induces senescence-like growth arrest with attenuated Ras-MAPK signaling in human cancer cells. Oncogene 2006;25:176-185.

-37 Chen WY, Wang DH, Yen RC, Luo J, Gu W, Baylin SB: Tumor suppressor HIC1 directly regulates SIRT1 to modulate p53-dependent DNA-damage responses. Cell 2005;123:437-448.

-38 Zhao W, Kruse JP, Tang Y, Jung SY, Qin J, Gu W: Negative regulation of the deacetylase SIRT1 by DBC1. Nature 2008;451:587-590.

-39 Yeung F, Hoberg JE, Ramsey CS, Keller MD, Jones DR, Frye RA, et al: Modulation of NF-kappaB-dependent transcription and cell survival by the SIRT1 deacetylase. EMBO J 2004;23:2369-2380.

40 Yuan J, Minter-Dykhouse K, Lou Z: A c-Myc-SIRT1 feedback loop regulates cell growth and transformation. J Cell Biol 2009;185:203-211.

41 Rusin M, Zajkowicz A, Butkiewicz D: Resveratrol induces senescence-like growth inhibition of U-2 OS cells associated with the instability of telomeric DNA and upregulation of BRCA1. Mech Ageing Dev 2009;130: 528-537.

42 Lin HY, Tang HY, Keating T, Wu YH, Shih A, Hammond D, et al: Resveratrol is pro-apoptotic and thyroid hormone is anti-apoptotic in glioma cells: both actions are integrin and ERK mediated. Carcinogenesis 2008; 29:62-69.

-43 Crew KD, Neugut AI: Epidemiology of gastric cancer. World J Gastroenterol 2006;12:354-362.

44 Hanahan D, Weinberg RA: Hallmarks of cancer: the next generation. Cell 2011;144:646-674.

-45 Ford J, Jiang M, Milner J: Cancer-specific functions of SIRT1 enable human epithelial cancer cell growth and survival. Cancer Res 2005;65:10457-10463.

-46 Chung S, Yao H, Caito S, Hwang JW, Arunachalam G, Rahman I: Regulation of SIRT1 in cellular functions: role of polyphenols. Arch Biochem Biophys 2010;501:79-90.

47 Audrito V, Vaisitti T, Rossi D, Gottardi D, D’Arena G, Laurenti L, et al: Nicotinamide blocks proliferation and induces apoptosis of chronic lymphocytic leukemia cells through activation of the p53/miR-34a/SIRT1 tumor suppressor network. Cancer Res 2011;71:4473-4483.

-48 Li Y, Backesjo CM, Haldosen LA, Lindgren U: Resveratrol inhibits proliferation and promotes apoptosis of osteosarcoma cells. Eur J Pharmacol 2009;609:13-18.

49 Pizarro JG, Verdaguer E, Ancrenaz V, Junyent F, Sureda F, Pallas M, et al: Resveratrol inhibits proliferation and promotes apoptosis of neuroblastoma cells: role of sirtuin 1. Neurochem Res 2011;36:187-194.

50 Evan GI, Vousden KH: Proliferation, cell cycle and apoptosis in cancer. Nature 2001;411:342-348. 